\title{
LETTER
}

\section{Effectiveness of inhaled furosemide for acute asthma exacerbation: a meta-analysis}

Ryota Inokuchi $i^{12^{*}+}$, Ai Aoki ${ }^{1,3,4 \dagger}$, Yuta Aoki ${ }^{1,4+}$ and Naoki Yahagi

As the effectiveness of beta-agonists for treating asthma attacks has been established, numerous other supportive treatments for asthma attacks have also been investigated, such as systemic glucocorticoids and magnesium. Among these additional therapies, inhaled furosemide is of particular interest; several studies have evaluated the effects of prophylactic inhaled furosemide in attenuating bronchoconstriction and asthma attacks. To determine the efficacy of inhaled furosemide during asthma attacks, we performed a systematic review using the MEDLINE, EMBASE, Web of Science, and Cochrane Library databases from their inception through 14 March 2014. A meta-analysis was conducted by calculating the standardized mean difference from each study and integrating these means using a random effects model. In addition, subanalyses were performed in the studies that evaluated the peak expiratory flow rate and the forced expiratory volume in 1 second.

We identified six studies using double-blinded, randomized control trial designs that evaluated inhaled furosemide in conjunction with standard treatments in patients experiencing asthma attacks [1-6] (Figure 1); a total of 78 patients received inhaled furosemide and 79 patients received a placebo (Tables 1 and 2). The mean age of patients ranged from 8.4 to 47 years $[3,6]$. In two studies, patients were administered $40 \mathrm{mg}$ inhaled furosemide [1,2]; in one study, patients were administered $20 \mathrm{mg}$ inhaled furosemide [6]; and in the three studies that recruited children, patients were administered either $1.0 \mathrm{mg} / \mathrm{kg}[4,5]$ or $10 \mathrm{mg} / \mathrm{m}^{2}$ [3] inhaled furosemide.

Integrating the standardized mean difference in each study, a random effects model showed that inhaled furosemide had a significant positive effect on asthma attacks $(Z=2.70 ; 95 \%$ confidence interval, 0.14 to 0.85 ; $P=0.007)$ with a negligible heterogeneity $\left(I^{2}=16.82\right)$ (Figure 2 and Table 3). Subanalyses of the studies reporting the peak expiratory flow rate $(Z=2.23 ; P=0.026$; $n=68 / 70$, inhaled furosemide/placebo) and the forced expiratory flow in 1 second $(Z=1.84 ; P=0.066 ; n=49 / 46$, inhaled furosemide/placebo) values confirmed the significant effectiveness of inhaled furosemide for asthma attacks (Table 3). Jackknife sensitivity analyses confirmed the replicability of these findings $(P<0.028)$ (Figure 3). No adverse events associated with furosemide inhalation were reported.

These results thus reveal a statistically significant improvement in airflow obstruction with no evident adverse events when inhaled furosemide was used as an adjunctive treatment for acute asthma exacerbation. The present study provides evidence supporting the addition of inhaled furosemide to conventional treatment in clinical situations.

* Correspondence: inokuchi-icu@h.u-tokyo.ac.jp

${ }^{\dagger}$ Equal contributors

'Department of Emergency and Critical Care Medicine, The University of

Tokyo Hospital, 7-3-1 Hongo, Bunkyo-ku, Tokyo 113-8655, Japan

2Department of Emergency Medicine, JR General Hospital, Yoyogi,

Shibuya-ku, Tokyo 151-8528, Japan

Full list of author information is available at the end of the article 


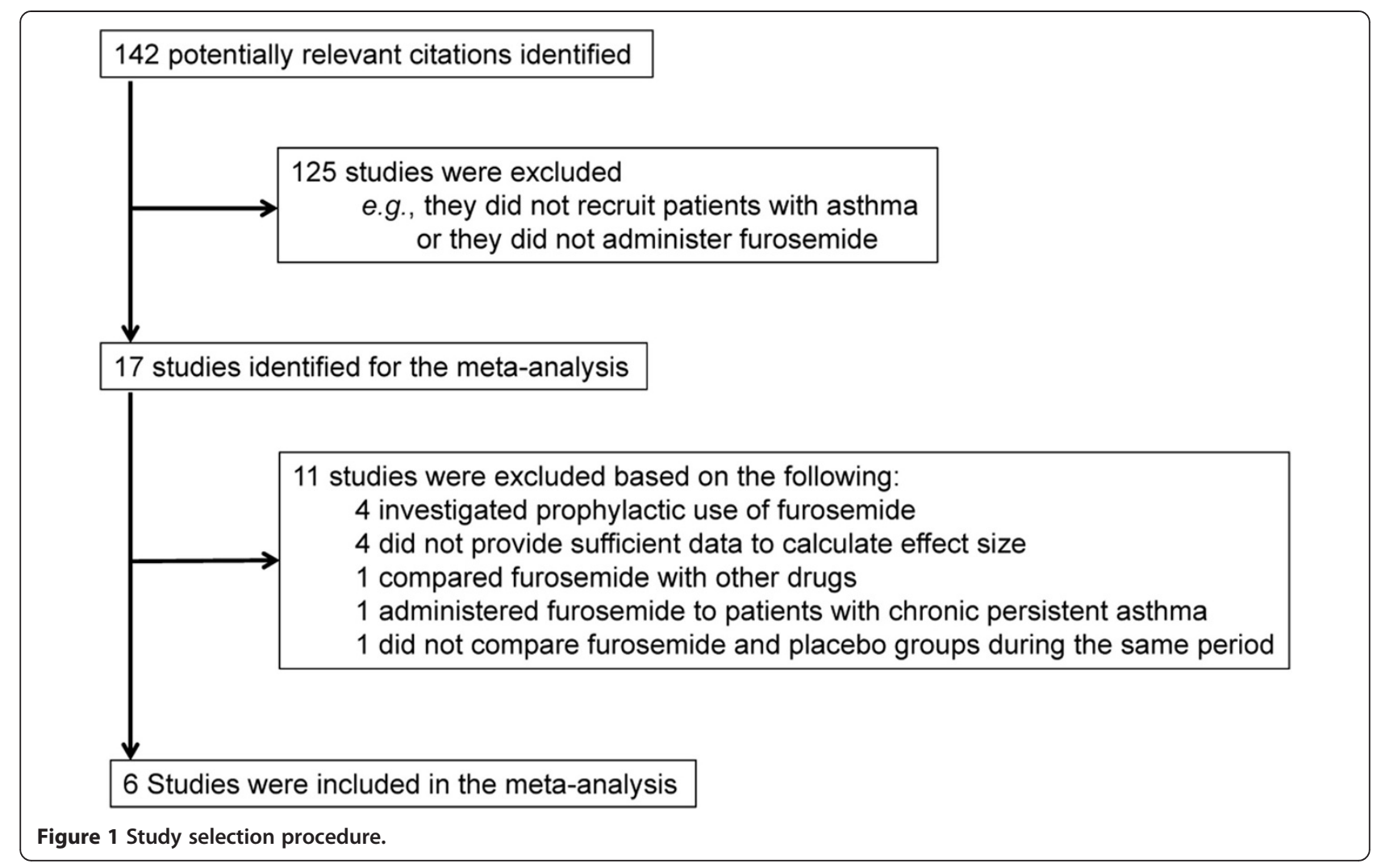


Table 1 Trial characteristics

\begin{tabular}{|c|c|c|c|c|c|c|c|c|c|c|c|c|c|c|}
\hline \multirow[b]{2}{*}{ Study (year) } & \multirow[b]{2}{*}{$\begin{array}{l}\text { Number of } \\
\text { randomized } \\
\text { patients }\end{array}$} & \multirow[b]{2}{*}{$\begin{array}{l}\text { Number of } \\
\text { patients } \\
\text { completing } \\
\text { study }\end{array}$} & \multicolumn{2}{|c|}{ Furosemide group } & \multicolumn{2}{|c|}{ Placebo group } & \multirow[b]{2}{*}{ Smoking } & \multirow[b]{2}{*}{ COPD } & \multirow[b]{2}{*}{$\begin{array}{l}\beta \text {-agonist } \\
\text { dose }\end{array}$} & \multirow[b]{2}{*}{$\begin{array}{l}\text { Inhaled } \\
\text { furosemide } \\
\text { dose }\end{array}$} & \multirow[b]{2}{*}{$\begin{array}{l}\text { Expiratory } \\
\text { airflow } \\
\text { assessment } \\
\text { time (minutes) }\end{array}$} & \multirow[b]{2}{*}{$\begin{array}{l}\text { Spirometry } \\
\text { measurement } \\
\text { used }\end{array}$} & \multirow[b]{2}{*}{ Severity } & \multirow[b]{2}{*}{$\begin{array}{l}\text { Hydrocortisone } \\
\text { (mg) }\end{array}$} \\
\hline & & & $\begin{array}{l}\text { Mean patient } \\
\text { age (years) }\end{array}$ & $\begin{array}{l}\text { Number of } \\
\text { patients } \\
\text { (male) }\end{array}$ & $\begin{array}{l}\text { Mean patient } \\
\text { age (years) }\end{array}$ & $\begin{array}{l}\text { Number of } \\
\text { patients } \\
\text { (male) }\end{array}$ & & & & & & & & \\
\hline $\begin{array}{l}\text { Alshehri and } \\
\text { colleagues, } \\
2005 \text { [5] }\end{array}$ & 39 & 39 & 8.4 & $19(11)$ & 8.5 & $20(9)$ & $N / A^{a}$ & $\mathrm{~N} / \mathrm{A}^{\mathrm{a}}$ & $0.15 \mathrm{mg} / \mathrm{kg}$ & $1.0 \mathrm{mg} / \mathrm{kg}$ & 30 & PEFR, FEV1.0 & Moderate & $\mathrm{N} / \mathrm{A}$ \\
\hline $\begin{array}{l}\text { González- } \\
\text { Sánchez } \\
\text { and colleagues, } \\
2002 \text { [4] }\end{array}$ & 20 & 20 & 9.8 & $10(7)$ & 10 & $10(5)$ & $N / A^{a}$ & $N / A^{a}$ & $0.15 \mathrm{mg} / \mathrm{kg}$ & $1.0 \mathrm{mg} / \mathrm{kg}$ & 30,60 & FEV1.0 & $\begin{array}{l}\text { Mild or } \\
\text { moderate }\end{array}$ & N/A \\
\hline $\begin{array}{l}\text { Nannini and } \\
\text { colleagues, } \\
1992 \text { [1] }\end{array}$ & 20 & 16 & 31 & $7(\mathrm{~N} / \mathrm{A})$ & 41 & $9(\mathrm{~N} / \mathrm{A})$ & N/A & N/A & $2.5 \mathrm{mg}$ & $40 \mathrm{mg}$ & 15,30 & PEFR & N/A & $\mathrm{N} / \mathrm{A}$ \\
\hline $\begin{array}{l}\text { Nuhoğlu and } \\
\text { colleagues, } \\
2006 \text { [3] }\end{array}$ & 32 & 32 & 8.6 & $16(8)$ & 8.4 & $16(12)$ & $N / A^{a}$ & $N / A^{a}$ & $0.15 \mathrm{mg} / \mathrm{kg}$ & $10 \mathrm{mg} / \mathrm{m}^{2}$ & N/A & PEFR & $\begin{array}{l}\text { Mild or } \\
\text { moderate }\end{array}$ & N/A \\
\hline $\begin{array}{l}\text { Ono and } \\
\text { colleagues, } \\
1997^{b} \text { [6] }\end{array}$ & 37 & 37 & 47 & $20(7)$ & 41 & $17(8)$ & N/A & Exclude & N/A & $20 \mathrm{mg}$ & 30,60 & PEFR, FEV1.0 & $\begin{array}{l}\text { Mild to } \\
\text { severe }\end{array}$ & 100 \\
\hline $\begin{array}{l}\text { Pendino and } \\
\text { colleagues, } \\
1998^{\mathrm{C}}[2]\end{array}$ & 42 & 42 & 38 & $6(\mathrm{~N} / \mathrm{A})$ & 34 & $8(\mathrm{~N} / \mathrm{A})$ & $\begin{array}{l}\text { Not }>10 \\
\text { pack-years }\end{array}$ & Exclude & $2.5 \mathrm{mg}$ & $40 \mathrm{mg}$ & 15,30 & PEFR & $\begin{array}{l}\text { Mild or } \\
\text { moderate }\end{array}$ & 300 \\
\hline
\end{tabular}

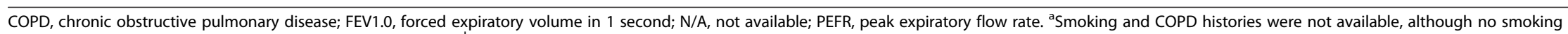
or COPD history was assumed because patients were children. ${ }^{\circ}$ Combination treatment in all trials was simultaneous administration of a beta-agonist plus furosemide, except for Ono and colleagues, in which patients in both groups received hydrocortisone succinate and aminophylline, followed 30 minutes later by either furosemide or placebo. 'Only subgroup data (pertaining to patients whose exacerbations lasted $<8$ hours) were available. 
Table 2 Trial results

\begin{tabular}{|c|c|c|c|c|c|c|c|c|c|c|c|c|c|c|c|c|}
\hline \multirow[b]{3}{*}{ Study (year) } & \multicolumn{8}{|c|}{ Furosemide } & \multicolumn{8}{|l|}{ Placebo } \\
\hline & \multicolumn{4}{|l|}{ PEFR } & \multicolumn{4}{|l|}{ FEV1.0 } & \multicolumn{4}{|l|}{ PEFR } & \multicolumn{4}{|l|}{ FEV 1.0} \\
\hline & $\begin{array}{l}\text { Baseline } \\
\text { airflow }\end{array}$ & SD & $\begin{array}{l}\text { Post-treatment } \\
\text { airflow }\end{array}$ & SD & Baseline airflow & SD & $\begin{array}{l}\text { Post-treatment } \\
\text { airflow }\end{array}$ & SD & $\begin{array}{l}\text { Baseline } \\
\text { airflow }\end{array}$ & SD & $\begin{array}{l}\text { Post-treatment } \\
\text { airflow }\end{array}$ & SD & $\begin{array}{l}\text { Baseline } \\
\text { airflow }\end{array}$ & SD & $\begin{array}{l}\text { Post-treatment } \\
\text { airflow }\end{array}$ & SD \\
\hline $\begin{array}{l}\text { Alshehri and colleagues, } \\
2005 \text { [5] }\end{array}$ & 59.0 & 22.0 & 84.9 & 14.0 & 58.5 & 14.5 & 80.2 & 13.9 & 57.2 & 25.4 & 80.7 & 17.4 & 56.7 & 17.3 & 77.8 & 19.1 \\
\hline \multirow{2}{*}{$\begin{array}{l}\text { Nuhoğlu and colleagues, } \\
2006 \text { [3] }\end{array}$} & 178 & 65.9 & 222 & 66.1 & $\mathrm{~N} / \mathrm{A}$ & $\mathrm{N} / \mathrm{A}$ & 183 & 51.7 & 218 & 60.3 & N/A & $\mathrm{N} / \mathrm{A}$ & & & & \\
\hline & $\begin{array}{l}\text { Baseline } \\
\text { airflow }\end{array}$ & SD & $\begin{array}{l}\text { Net airflow } \\
\text { improvement } \\
\text { above baseline }\end{array}$ & SD & Baseline airflow & SD & $\begin{array}{l}\text { Net airflow } \\
\text { improvement } \\
\text { above baseline }\end{array}$ & SD & $\begin{array}{l}\text { Baseline } \\
\text { airflow }\end{array}$ & SD & $\begin{array}{l}\text { Net airflow } \\
\text { improvement } \\
\text { above baseline }\end{array}$ & SD & $\begin{array}{l}\text { Baseline } \\
\text { airflow }\end{array}$ & SD & $\begin{array}{l}\text { Net airflow } \\
\text { improvement } \\
\text { above baseline }\end{array}$ & SD \\
\hline $\begin{array}{l}\text { González-Sánchez and } \\
\text { colleagues, } 2002 \text { [4] }\end{array}$ & N/A & N/A & 0.820 & 0.460 & 0.910 & 0.067 & N/A & $\mathrm{N} / \mathrm{A}$ & 0.850 & 0.340 & 0.980 & 0.078 & & & & \\
\hline $\begin{array}{l}\text { Nannini and colleagues, } \\
1992 \text { [1] }\end{array}$ & 147 & 68.0 & 269 & 89.7 & $\mathrm{~N} / \mathrm{A}$ & N/A & 234 & 82.0 & 316 & 56.2 & N/A & N/A & & & & \\
\hline $\begin{array}{l}\text { Ono and colleagues, } \\
1997 \text { [6] }\end{array}$ & 171 & 20.0 & 205 & 45.1 & 1.18 & 0.13 & & 178 & 25.0 & 198 & 21.3 & 1.32 & 0.74 & N/A & & \\
\hline $\begin{array}{l}\text { Pendino and colleagues, } \\
1998 \text { [2] }\end{array}$ & 200 & 71.0 & 426 & 98.0 & $\mathrm{~N} / \mathrm{A}$ & N/A & 209 & 68.0 & 337 & 73.2 & $\mathrm{~N} / \mathrm{A}$ & N/A & & & & \\
\hline
\end{tabular}




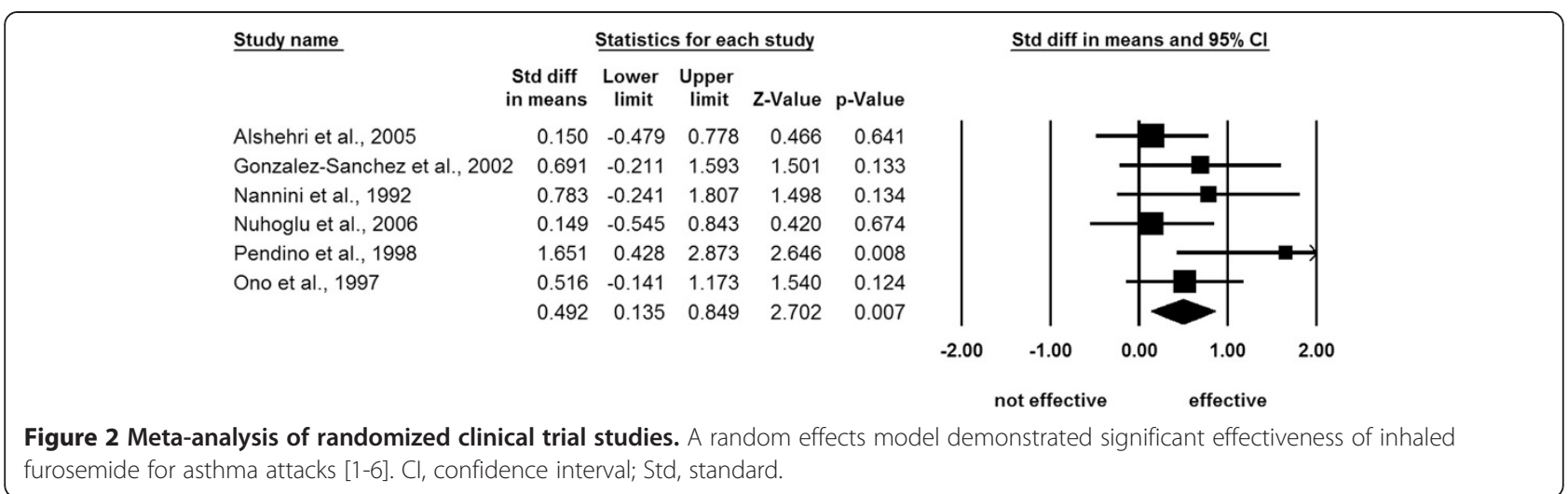

Table 3 Meta-analyses of randomized controlled trials

\begin{tabular}{|c|c|c|c|c|c|c|c|c|}
\hline & Number of studies & Furosemide group $(n)$ & Placebo group (n) & Lower $95 \% \mathrm{Cl}$ & Upper $95 \% \mathrm{Cl}$ & $Z$ value & $P$ value & $P^{2}$ \\
\hline Whole studies & 6 & 78 & 79 & 0.14 & 0.85 & 2.70 & 0.007 & 16.8 \\
\hline PEFR & 5 & 68 & 70 & 0.058 & 0.90 & 2.23 & 0.026 & 30.4 \\
\hline FEV1.0 & 3 & 49 & 46 & -0.027 & 0.83 & 1.84 & 0.066 & 8.16 \\
\hline
\end{tabular}

$\mathrm{Cl}$, confidence interval; FEV1.0, forced expiratory volume in 1 second; PEFR, peak expiratory flow rate.

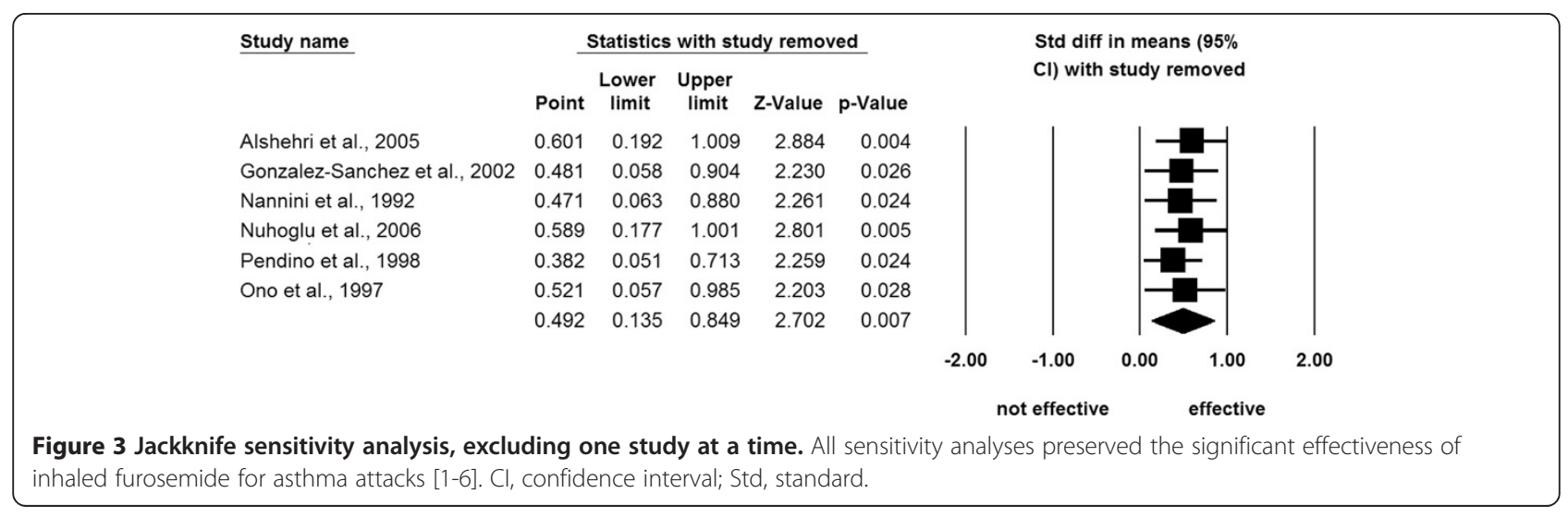




\section{Competing interests}

The authors declare that they have no competing interests.

\section{Authors' contributions}

$\mathrm{RI}$ and AA drafted the initial manuscript. YA contributed to the manuscript composition. RI and AA both independently screened the studies. YA performed the statistical analyses. NY critically reviewed the manuscript. All authors provided written consent for publication. All authors read and approved the final manuscript.

\section{Acknowledgements}

The authors are grateful to Dr Masao Iwagami (The London School of Hygiene \& Tropical Medicine, London, UK) for his assistance.

\section{Author details}

'Department of Emergency and Critical Care Medicine, The University of Tokyo Hospital, 7-3-1 Hongo, Bunkyo-ku, Tokyo 113-8655, Japan.

${ }^{2}$ Department of Emergency Medicine, JR General Hospital, Yoyogi, Shibuya-ku, Tokyo 151-8528, Japan. ${ }^{3}$ Department of Psychiatry, Tokyo Metropolitan Health and Medical Treatment Corporation, Ebara Hospital, Ota-ku, Tokyo 145-0065, Japan. ${ }^{4}$ Department of Neuropsychiatry, Graduate School of Medicine, The University of Tokyo, Bunkyo-ku, Tokyo 113-8655, Japan.

\section{Published online: 24 November 2014}

\section{References}

1. Nannini L, Pendino J, Molfino N, Slutsky A: Inhaled furosemide and salbutamol in acute asthma. Am Rev Respir Dis 1992, 145:422.

2. Pendino JC, Nannini LJ, Chapman KR, Slutsky A, Molfino NA: Effect of inhaled furosemide in acute asthma. J Asthma 1998, 35:89-93.

3. Nuhoğlu C, Yaşar Kiliç M, Ceran O: Effectiveness of nebulized furosemide added to nebulized salbutamol in children with acute asthma. Allergol Immunopathol (Madr) 2006, 34:54-58.

4. González-Sánchez R, Trujillo-Hernández B, Huerta M, Vásquez C, Trujillo X: Furosemide plus albuterol compared with albuterol alone in children with acute asthma. Allergy Asthma Proc 2002, 23:181-184.

5. Alshehri M, Almegamesi T, Alfrayh A: Efficacy of nebulized furosemide in children with moderate attack of asthma. West Afr J Med 2005, 24:246-251.

6. Ono $Y$, Kondo $T$, Tanigaki $T$, Ohta $Y$ : Furosemide given by inhalation ameliorates acute exacerbation of asthma. J Asthma 1997, 34:283-289.

doi:10.1186/s13054-014-0621-y

Cite this article as: Inokuchi et al:: Effectiveness of inhaled furosemide

for acute asthma exacerbation: a meta-analysis. Critical Care 2014 18:621. 